1990- Catálogo de las Gramíneas malacitanas. Acta Bot. Malacitana 15: 91-123.

CLAYTON, W.D. -1980- Pennisetum. In: T.G. Tutin \& al. -eds.- Flora Europaea 5: 264. Cambridge.

CRESPO, M.B. -1996- Dos neófitos novedosos para la flora de Valencia. Fl. Montiber. 4: 8384.

CRESPO, M.B., M.L. MANSO y G. MATEO 1990- Pennisetum setaceum (Poaceae), especie nueva para el continente europeo. Anales Jard. Bot. Madrid 47(1): 260 (1990)

OAKES, A.J. -1990- Ornamental grasses and grasslike plants. New York.

SÁNCHEZ, P., M.A. CARRIÓN, A. HERNÁNDEZ, J.B. VERA y J.A. LÓPEZ 2003- Notas corológicas y nomenclaturales para la flora del sureste ibérico. Anales Biol., Fac. Biol., Univ. Murcia 25: 109-112.
SANZ-ELORZA, M., E.D. DANA y E. SOBRINO -2005- Aproximación al listado de plantas vasculares alóctonas invasoras reales y potenciales en las islas Canarias. Lazaroa 26: 55-66.

SERRA, J.A. y M.B. CRESPO -1995- Adiciones a la flora alicantina. Acta Bot. Malacitana 20: 284-290.

SHERIF, A.S. \& M.A. SIDDIQI -1988- Poaceae. In: A.A. El-Gadi -ed.- Flora of Libya 145: 1356. Trípoli.

WATSON, L. \& M.J. DALLWITZ -1992- The grass genera of the World. Cambridge.

Aceptado para su publicación en octubre de 2006

Dirección de los autores: Departamento de Botánica, Ecología y Fisiología Vegetal, Universidad de Córdoba, Campus de Rabanales, Edif. J. Celestino Mutis, ctra. de Madrid km. 396, 14071-Córdoba.

\title{
117. NOVEDADES FLORÍSTICAS EN EL PARQUE NACIONAL DE DOÑANA (SW ESPAÑA)
}

Rocío FERNÁNDEZ ZAMUDIO, Santos CIRUJANO, Iván NIETO GIL, M. Dolores COBO, Arturo SOUSA MARTÍN y Pablo GARCÍA MURILLO

New floristic records for Doñana National Park (Sw Spain)

Palabras clave. Flora, macrófitos acuáticos, Chara, Azolla, especies amenazadas, especies invasoras, Doñana, humedales.

Key words. Flora, aquatic macrophytes, Chara, Azolla, threatened species, invasive species, Doñana, wetlands.

Las novedades que se presentan son el resultado de los estudios de caracterización florística que se están realizando en los humedales de Doñana. Por un lado se han recolectado diversos macrófitos acuáticos de los que existen pocas referencias a nivel peninsular, lo que pone en evidencia la importancia que tienen estos humedales del 
SW europeo para el mantenimiento de la flora acuática e hidrófila de esta parte de Europa. Por otro lado, se citan algunas plantas terrestres singulares. Finalmente, se refiere la presencia de plantas exóticas invasoras cuya aparición hay que ligarla a las perturbaciones antrópicas que inciden en diversos lugares de este espacio natural protegido.

\section{CHLOROPHYTA}

\section{Chara fragilis Desv.}

[Chara globularis var. globularis f. globularis (Thuill.) R. D. Wood]

Carófito cosmopolita de aguas poco mineralizadas, que vive en aguas estancadas o con ligera corriente (Comelles, 1985). Existen dos citas previas en la provincia de Huelva, que corresponden a San Silvestre de Guzmán. Las recolecciones en diferentes enclaves de Doñana amplían su área de distribución en el SW peninsular.

HUELVA: Almonte, P. N. de Doñana, laguna de Montalban, 29SQB2912, 09/03/04, Fdez Zamudio, MA-Algae 10957; ídem, navazo de la Higuera, 29SQA3281, 27/04/04, Fdez. Zamudio, García Murillo \& Cirujano, MA-Algae 10955; idem, 07/04/04, Fdez. Zamudio, MA-Algae 10956.

Chara vulgaris var. contraria (A. Braun ex Kütz.) Moore

Carófito cosmopolita que crece en una amplia variedad de hábitat, siendo más frecuente en aguas con pH elevados (Moore, 1986). Las primeras citas de este taxon en la provincia de Huelva se deben a Sánchez (1984) que lo menciona de diversos enclaves de la sierra de Huelva. Nuestras recolecciones de Doñana confirman la presencia de este taxón en los cuerpos de agua del litoral onubense, donde no se conocía anteriormente.

HUELVA: Almonte, Parque Nacional de Doñana, navazo de la Higuera, 29SQA3281, $27 /$ 04/04, Cirujano, MA-Algae 10349; idem, 20/02/ 04, Fdez. Zamudio \& García Murillo, MA-Algae 10954.
Chara vulgaris var. longibracteata (Kütz) J. Groves \& Bullock-Webster

Variedad propia de pequeños cuerpos de agua alcalinos y estacionales sobre sustratos limosos (Moore, 1986). Nuestra cita en Doñana es la primera para la provincia de Huelva.

HUELVA: Almonte, Doñana, navazo de la Higuera, 29SQA3281, 20/02/04, Fdez Zamudio \& García Murillo, MA-Algae 10953.

\section{PTERIDOPHYTA}

\section{Azolla filiculoides Lam.}

\section{(= Azolla caroliniana (Willd.)}

Pteridófito acuático procedente del Nuevo Mundo, propio de aguas tranquilas, dulces o subsalinas, y ricas en nutrientes. Su presencia en la Península Ibérica está contrastada desde 1920, cuando fue recolectada en los arrozales de la desembocadura del río Sado, en Portugal. Desde entonces se ha extendido por distintos humedales de España y Portugal. Se han encontrado importantes poblaciones en los humedales de Beira Litoral, desembocaduras del Tajo, Ebro, Llobregat, y un buen número de arroyos, ríos, charcas y embalses del Alentejo, W de Extremadura, Salamanca, Ciudad Real, Madrid, etc. En los lugares donde se desarrolla llega a cubrir extensas superficies, reduce o impide la penetración de la luz en el agua, disminuye los niveles de oxígeno disuelto y desplaza a los macrófitos acuáticos sumergidos, nativos de los humedales que coloniza. En la provincia de Huelva fue citada por primera vez en el Estero de Domingo Rubio, término de Moguer (Sánchez Gullón \& Macias, 2000), y en el año 2001 técnicos del Parque Nacional de Doñana la identificaron en los humedales de Doñana (García Murillo et al., 2004). Durante la primavera del año 2004, sus poblaciones cubrieron una gran parte de la marisma del Parque Nacional, observándose también en algunos cuerpos de agua sobre las arenas, como la laguna del Pinar de San Agustín. Asimismo, García Murillo et al. (2005) la encotraron ese mismo año en los canales de riego de la colonia Monte Algaida, en Sanlúcar de Barrameda (Cádiz), situados frente al Parque Nacional de Doñana.

HUELVA: Almonte, Parque Nacional de Doñana, Caño de Soto Grande, 29SQB2408, 20/ 
01/06, Fdez. Zamudio, SEV 215738; ídem, marisma frente a la Casa de los Carabineros, 29SQB3989, 27/04/4, Fdez. Zamudio, SEV 215741; ídem, laguna del Hondón, 29SQA2990, 01/04/05, Fdez. Zamudio, SEV 215174; ídem, 21/ 01/06, Fdez. Zamudio, SEV 215744; ídem, laguna de las Pajas, 29SQA2595, 16/06/04, Fdez. Zamudio, SEV 215746; ídem, lucio del Caballero, 29SQA2893, 19/01/06, Fdez. Zamudio, SEV 215743; ídem, lucio del Membrillo, 29SQA3586, 23/04/04, Fdez. Zamudio, SEV 215739; ídem, lucio de los Ánsares, 29SQA4192, 15/06/04, Fdez. Zamudio, SEV 215740; ídem, vera junto a las Pajareras, 29SQA2897, 25/03/04, Fdez. Zamudio, SEV 215736; ídem, zacallón de la laguna del pinar de San Agustín, 29SQA2797, 13/ 04/04, Fdez. Zamudio, SEV 215742.

\section{Marsilea strigosa Willd.}

Pteridófito característico de lugares encharcados temporalmente. Su presencia en Huelva había sido indicada anteriormente del término de Niebla (Silvestre, 2000). La nueva localidad amplía su área de distribución. Aparece en la "Lista roja de la flora vascular de Andalucía" (Cabezudo et al., 2005) con la categoría de VU (especies vulnerables)

HUELVA: Almonte, Parque Nacional de Doñana, Soto de los Guayules, 29SQB2209, 10/ 06/04, Fdez. Zamudio, SEV 215173.

\section{SPERMATOPHYTA}

\section{Callitriche obtusangula Le Gall}

Macrófito acuático sumergido, cuya presencia para la provincia de Huelva se señala aquí por primera vez. Esta planta, frecuente en las aguas dulces y subsalinas de Doñana, fue relacionada anteriormente con C. platycarpa Kütz in Reichemb, siendo ésta una especie propia de Europa Central y rara en la Península Ibérica, de la que se distingue, C. obtusangula, por sus frutos más largos que anchos, entre otros caracteres. Aparece en la "Lista roja de la flora vascular de Andalucía" (Cabezudo et al., 2005) con la categoría de DD (datos insuficientes).

HUELVA. Almonte, Parque Nacional de Doñana, 24/04/1983, Smit, SEV106882; ídem, Casa del Martinazo, 24/04/1983, Smit, SEV106881, MA465832; ídem, marisma del
Rocío, 20/05/1982, Valdés, SEV112057; Reserva Biológica de Doñana, marisma, 09/03/1973, Cabezudo, SEV17074 ; ídem, en la vera entre el Palacio y Martinazo, 29SQA2899, 08/04/84, García Murillo \& Smit, SEV 215737; ídem, rincón de la laguna del Sopetón, 29SQA2793, 20/ 01/06, Fdez. Zamudio, SEV 215745

Halopeplis amplexicaulis (Vahl) Ung. Stemb. ex Ces., Pass.\& Gilbelli

Terófito propio de suelos salinos, raro en la Península Ibérica. Se distribuye (Blanché, 1990) de forma disyunta por el Algarve, Alicante (E1 Hondo de Elche-Crevillente), litoral de Cádiz, Teruel (los Monegros) y Málaga (laguna de Fuente de Piedra). Aparece en la "Lista roja de la flora vascular de Andalucía" (Cabezudo et al., 2005) con la categoría de NT (especies casi amenazadas).

HUELVA: Almonte, Salinas del Parque Nacional Doñana, 29SQA3584, 29/03/1994, Urdiales, herbario Parque Nacional Doñana; ídem, saladar mareal al sur de las salinas, 29SQA3584, 29/04/1996, Urdiales, herbario Parque Nacional Doñana; ídem Zona mareal al sur de las salinas, 29SQA3583, 30/04/1996, Urdiales, herbario Parque Nacional Doñana; ídem Motaña del río, 29SQA3582, 15/03/06, Nieto Gil SEV 215749.

Hymenolobus procumbens (L.) Nutt. in Torr. \& A. Gray subsp. procumbens

Terófito de suelos salinos. Aparece en paciles (pequeñas elevaciones del terreno en la marisma) en la base de Arthrocnemum macrostachyum. No ha sido citada para la provincia de Huelva.

HUELVA: Almonte, Parque Nacional de Doñana, Montaña del Río, extremo sur,29SQA3581, 11/03/05, Nieto Gil \& García Murillo, SEV 215166.

\section{Marrubium vulgare $\mathrm{L}$.}

Caméfito de carácter nitrófilo del que no se han hallado citas anteriores en zonas de marisma. Se ha encontrado en una veta conchífera que aflora en medio de la marisma salada. Las únicas referencias de este taxon para la provincia de Huelva proceden de la Sierra de Aracena (Rivera \& Cabezudo, 1985); en Sevilla la cita más cercana corresponde al término de Huévar (Barras de Aragón, 1898). 
SEVILLA: Aznalcazar, Parque Nacional de Doñana, veta entre Las Nuevas y la casa del Cherry, 29SQA3992, 14/05/05, Nieto Gil \& García Murillo, SEV 215163.

Puccinellia fasciculata (Torrey) E. P. Bicknell.

Hierba terrestre perenne propia de suelos salinos. No se ha encontrado ninguna cita para el territorio estudiado. Aparece en la "Lista roja de la flora vascular de Andalucía" (Cabezudo et al., 2005) con la categoría de VU (especies vulnerables)

SEVILLA: Aznalcazar, Doñana, al sur del caño de Buen Tiro, 29SQA421923, 14/05/05, Nieto Gil \& García Murillo, SEV 215165.

Wolffia arrhiza (L.) Horkel ex Wimmer

Acropleustófito que vive en aguas tranquilas de conductividad baja y elevadas concentraciones de nutrientes. Aparece en la "Lista roja de la flora vascular de Andalucía" (Cabezudo et al., 2005) con la categoría de EN (especies en peligro). Su presencia en el Parque Nacional de Doñana fue advertida por García Murillo (2000), aunque sin precisar la localización, y recientemente por Galán de Mera \& Castroviejo (2005). No obstante, al ser una especie de la que conocen pocas localizaciones, pensamos que resulta de gran interés citar esta nueva localidad, bastante diferente de la indicada por Galan de Mera \& Castroviejo (2005). Se ha encontrado en un "zacallón", que es un abrevadero artificial, más o menos profundo, situado sobre arenas, con el nivel freático próximo a la superficie. Mediante los “zacallones", en Doñana, se asegura el abastecimiento de agua a los animales durante la época estival. Se trata de uno de los escasos habitat de Doñana que mantienen agua durante todo el año y, por tanto, sirven de refugio a especies que precisan esta condición.

HUELVA: Almonte, Parque Nacional de Doñana, zacallón Regianes, 29SQA3280, 29/06/ 05, Fdez. Zamudio \& García Murillo, SEV 215167.

\section{Xanthium spinosum L.}

Hierba terrestre anual, originaria de América del Sur. En Europa se encuentra en suelos degradados y con altos contenidos en materia orgánica. En Doñana se ha observado sobre suelos arenosos frescos con claros indicios de eutrofia. En Huelva fue citada por primera vez para la Sierra de Aracena por Rivera \& Cabezudo (1985).

HUELVA: Almonte, Reserva Biológica de Doñana, borde de marisma próxima a las Pajareras, 29SQA2797, 28/06/05, Fdez. Zamudio \& García Murillo, SEV 215172.

AGRADECIMIENTOS. Trabajo realizado dentro del Proyecto «Doñana 2005» del Ministerio de Medio Ambiente. Los autores quieren agradecer al personal del Parque Nacional de Doñana, Parque Natural de Doñana y Reserva Biológica de Doñana la colaboración, la ayuda y las facilidades que en todo momento han disfrutado al realizar visitas y herborizaciones.

\section{BIBLIOGRAFÍA}

BARRAS DE ARAGÓN, F. DE LAS -1898- Datos para la flórula sevillana. Actas Sociedad Española de Historia Natural 27(5): 109-112.

BLANCHÉ C. -1990- Halopeplis Bunge ex Ung. Sternb. En Castroviejo, S. et al. (Eds.). Flora Iberica 2: 522-524. CSIC. Madrid.

CABEZUDO, B., S. TALAVERA, G. BLANCA, C. SALAZAR, M. CUETO, B. VALDÉS, J. E. HERNÁNDEZ BERMEJO, C. M. HERRERA, C. RODRÍGUEZ HIRALDO y D. NAVAS 2005- Lista Roja de la flora vascular de Andalucía. Consejería de Medio Ambiente, Junta de Andalucía. Sevilla. 126 pp.

COMELLES, M. -1985-Clave de identificación de las especies de carófitos de la Península Ibérica. Asociación española de Limnología. Universidad de Barcelona. 35 pp.

GALÁN DE MERA, A. y S. CASTROVIEJO 2005- Acerca del tratamiento de lemnaceae en flora iberica. Acta Bot. Malacitana 30: 248-249.

GARCÍA MURILLO, P. -2000- Wolffia arrhiza (L.) Horkel ex Wimmer. En Blanca, G. \& al. (Eds.) Libro Rojo de la Flora Amenazada de Andalucía. Tomo II. Especies vulnerables: 373-375. Consejería de Medio Ambiente, Junta de Andalucía. Sevilla.

GARCÍA MURILlO, P., M. D. COBO, E. SÁNCHEZ GULLÓN y H. GARRIDO -2004Una planta americana invade Doñana. 
Quercus 218: 46-47.

GARCÍA MURILLO, P., E. DANA SÁNCHEZ y

C. RODRÍGUEZ HIRALDO -2005- La lechuga de agua amenaza con invadir Doñana. Quercus 232: 36-37.

MOORE, J.A. -1986- Charophytes of Great Britain and Ireland. B.S.B.I. Handbook $\mathrm{n}^{\circ}$ 5. Botanical Society of the British Isles. London. $141 \mathrm{pp}$.

RIVERA, J. y B. CABEZUDO -1985- Aportaciones al conocimiento florístico de la Sierra de Aracena (Huelva, España). Acta Bot. Malacitana 10: 61-78.

SÁNCHEZ, P. M. -1984- Contribución al conocimiento del género Chara en Andalucía. Acta Bot. Malacitana 9: 79-84.

SÁNCHEZ GULLÓN, E. y M. J. MACIAS FUENTES -2000- Nuevos pteridófitos de la provincia de Huelva. I. Acta Bot. Malacitana 25: 229-230.
SILVESTRE, S. -2000- Marsilea strigosa Willd. En Blanca, G. \& al. (Eds.). Libro Rojo de la Flora Amenazada de Andalucía. Tomo II. Especies vulnerables: 232-234. Consejería de Medio Ambiente, Junta de Andalucía. Sevilla.

Aceptado para su publicación en julio de 2006

Dirección de los autores. R. FERNÁNDEZ ZAMUDIO, I. NIETO GIL, , A. SOUSA MARTÍN y P. GARCÍA MURILLO: Dpto. Biología Vegetal y Ecología. Facultad de Farmacia. Universidad de Sevilla. Apdo. 1036. 41080 Sevilla. España; S. CIRUJANO: Real Jardín Botánico (CSIC). Plaza de Murillo 2. 28014 Madrid. España; D. COBO: Parque Nacional de Doñana. Centro Administrativo del Acebuche. 21730-Almonte (Huelva). España.

118. VICIA SUBERVIFORMIS MAIRE, NUEVA ESPECIE PARA LA FLORA EUROPEA Carlos ROMERO ZARCO y Julio PEÑAS DE GILES

Vicia suberviformis, new specie for the European Flora

Palabras clave. Leguminosae, Vicia, flora europea, Granada, Marruecos.

Key words. Leguminosae, Vicia, European flora, Granada, Morocco.

Durante la revisión del material del Herbario de la Universidad de Granada para el proyecto «Flora de Andalucía Oriental» se han encontrado dos pliegos de una especie de arvejilla que se daba por endémica de Marruecos. Se trata de Vicia suberviformis Maire, perteneciente a un grupo de especies de flores pequeñas de la sección Cracca afines a $V$. vicioides (Desf.) Cout. que tienen en el Sur de la Península Ibérica y NW de África su centro de origen. La especie fue inicialmente descrita de Tamanar, en el W de Marruecos, región de Haha (Maire, 1923) y posteriormente citada también en el Rif por Sennen \& Mauricio (1933).

Los dos únicos ejemplares españoles 